\title{
Large Eddy Simulation of Bluff Body Stabilized Turbulent Premixed Flame
}

\author{
Nicolas Moisés Cruz Salvador, Márcio Teixeira de Mendonça², Wladimyr Mattos da Costa Dourado²
}

\begin{abstract}
A turbulent reacting flow in a channel with an obstacle was simulated computationally with large eddy simulation turbulence modeling and the $X i$ turbulent combustion model for premixed flame. The numerical model was implemented in the open source software OpenFoam. Both inert flow and reactive flow simulations were performed. In the inert flow, comparisons with velocity profile and recirculation vortex zone were performed as well as an analysis of the energy spectrum obtained numerically. The simulation with reacting flow considered a pre-mixture of propane $\left[\mathrm{C}_{3} \mathrm{H}_{8}\right]$ and air such that the equivalence ratio was equal to 0.65 , with a theoretical adiabatic flame temperature of 1,800 K. The computational results were compared to experimental ones available in the literature. The equivalence ratio, inlet flow velocity, pressure, flame-holder shape and size, fuel type and turbulence intensity were taken from an experimental set up. The results shown in the present simulations are in good agreement with the experimental data.
\end{abstract}

KEYWORDS: Computational fluid dynamics, Reacting flow, Large eddy simulation, Combustion modeling.

\section{INTRODUCTION}

In turbulent reactive flow simulations, computational models have achieved a great development in recent years with the improvement of computer power. This development allowed more accurate solution of problems such as the instability caused by the turbulence in combustion chambers of rocket engines, gas turbines, turbojet afterburners, ramjets and scramjets. These models have been used to study bluff body stabilized flames, which allow combustion devices to operate at very high free stream velocities. Advanced afterburner design methods have been discussed by Lovett et al. (2004), who outlined the fundamental combustion sciences and engineering challenges that need to be addressed. Among other problems, Lovett et al. (2004) highlighted the requirements for flame stabilization and combustion dynamics. These authors discuss the need for advanced design methodologies and tools, and they stress the limitations of existing computational models to capture the physics of those phenomena.

In turbulent combustion, the behavior of the turbulent flame front is predominantly dictated by the turbulence (Peters, 2000). Combustion instability is also directly related to turbulence (Weller, Marooney and Gosman, 1990). Therefore, it becomes mandatory in numerical simulations to use turbulence models that reproduce these dynamic processes, which are mainly produced by large scale turbulence. Many researchers have used Reynolds Averaged Navier Stokes (RANS) and eddy viscosity turbulence models to simulate reactive flows behind bluff bodies. However, important discrepancies were observed due to shortcomings in the RANS methodology, especially in complex flows with circular obstacle (Saghafian et al.,

\footnotetext{
1.Instituto Nacional de Pesquisas Espaciais - São José dos Campos/SP - Brazil 2.Instituto de Aeronáutica e Espaço - São José dos Campos/SP - Brazil Author for correspondence: Marcio Teixeira de Mendonça | Instituto de Aeronáutica e Espaço | Praça Marechal Eduardo Gomes, 50 - Vila das Acácias CEP 12.228-904 São José dos Campos/SP - Brazil | E-mail: marciomtm@iae.cta.br

Received: 22/06/12 | Accepted: 13/12/12
} 
2003; Frendi, Skarath and Tosh, 2004) and triangular one (Bai and Fuchs, 1994; Dourado, 2003; Eriksson, 2007). Significant differences in results were found for the turbulent velocity, integral length scale and turbulent viscosity distribution between RANS models. These differences affect the computation of flame front diffusion, which is underpredicted. The Kelvin-Helmholtz effects behind the obstacle are not captured well and the length of the recirculation zone as well as the turbulent flame speed are not recovered.

Experimental studies were conducted by Sjunesson, Henriksson and Lofstrom (1992) and Sjunnesson, Olovsson and Sjoblom (1991) at Volvo (Sweden). The Volvo test rig had an equilateral triangular bluff body with a blockage of $33 \%$ and a Reynolds number $(R e)$ based on the inlet velocity and two times the channel height of 204,000. For this $R e$, the Strouhal number (St) observed was 0.417 . The premixed gases were air and propane at equivalence ratios of 0.65 and 0.85 . The Damköhler number $(\mathrm{Da})$ was 10 and the Karlovitz number $(\mathrm{Ka})$ was 4 . The resulting flame for these conditions was on the thickened-wrinkled flame regime of the Borghi diagram rather than in the wrinkled flame regime. Sanquer (1998) also presented experimental results for premixed flame stabilized by a triangular prismatic bluff body at the University of Poitier (France). The blockage in his experiments was also $33 \%$ for a $R e$ in the range of 6,690 to 23,150 , much lower than the Re on the Volvo experiments. The St for the lower Re experiment was 0.276. Therefore, the vortex shedding characteristics and the turbulent scales (integral and Kolmogorov) were significantly different from the Volvo experiment. Sanquer's results will be discussed and compared to the present numerical simulations on the following sections. Specifically, the present simulations correspond to Sanquer's experiment that falls on the Borghi diagram where $D a<1$ and $K a>1$ corresponding to the thin-wrinkled flame region.

These and other experimental results (Cheng, 1984, Cheng, Shepherd and Gokalp, 1989, Cheng and Shepherd, 1991, Kiel et al., 2007, Chaudhuri et al., 2011) describe the interaction between the turbulent structures and the flame. Numerical simulations of turbulent combustion must rely on models that are able to capture the complex turbulent vortical structures and this explains why results obtained with RANS models are less accurate. In order to improve numerical simulations, turbulent combustion models can be ported from RANS models to Large Eddy Simulation (LES) models, which are known to capture the large scale turbulent structures. This approach does not always result in more accurate simulations. It is necessary to identify those models that have the best results and show greater potential for future improvements when used in LES.

A number of investigators have used LES to simulate reactive flows with bluff body flame holders. Porumbel and Menon (2006), Akula, Sadiki, and Janicka (2006), Ge et al. (2007), Park and Ko (2011) and Manickam et al. (2012) simulated the Volvo experiment (Sjunnesson, Olovsson and Sjoblom, 1991). Porumbel and Menon (2006) have simulated bluff body flows with the Linear Eddy Mixing (LEM) LES based on the model proposed by Kerstein (1989) and developed into a sub-grid model by Menon et al. (1993) for premixed combustion. They discussed the differences between their model and the Eddy Breakup model (LES-EBU) and which of the two is best to represent the physics of the reacting flow behind an obstacle besides the ability to resolve the turbulent eddies that wrinkle the flame front. This model is specially adequate for high turbulent intensities where the chemical time is considered infinitely small, corresponding to a $D a$ much greater than 1 . Their conclusions were confirmed by experimental results presented by Chaudhuri et al. (2011).

Akula, Sadiki, and Janicka (2006) also performed LES simulations with a flame surface density formulation adapted from Boger and Veynante (2000). This model includes the resolved progress variable in the transport equations and the flame wrinkling model avoids unrealistic detachment of the flame from the flame holder. The compressible model presented by Tabor and Weller (2004), along with a similar progress variable approach, is also able to capture the flame wrinkling and thus capture the physics as proposed by Porumbel and Menon (2006). The model of Tabor and Weller (2004) is used in the present simulations.

Park and Ko (2011) used a dynamic sub-grid scale combustion model based on the G-equation that describes the flame front propagation (Peters, 2000). The G-equation is a model for the chemical species conservation equations. In this model, a new LES dynamic sub-grid combustion model is introduced along with a new turbulent flame speed model based on the sub-grid turbulent diffusivity. These models are intended to better represent the flame characteristics. Park and Ko (2011) present comparisons between non-reacting and reacting flows and discuss the effect of chemical reaction on the development of the wake behind the triangular bluff body. They show that their model is able to capture the stabilization of the Karman vortex street 
behind the obstacle due to combustion. The results for the dynamic model show better agreement with experimental results when compared with the Smagorinsky model. The unsteady flow field was captured with good accuracy and the temperature and reaction rate profiles are well capture by both models, showing that the two combustion models tested are reasonable for the simulation of pre-mixed combustion behind a bluff body.

Manickam et al. (2012) used an algebraic flame surface wrinkling (AFSW) reaction model based on the progress variable approach. Inert and reacting cases were analysed and compared with experimental results and with another well validated turbulent flame speed closure model denominated Turbulent Flame Speed Closure (TFC) (Zimont and Lipatnikov, 1995). For the non-reacting test case, they found a shedding frequency equal to $122 \mathrm{~Hz}$, while the experimental measured frequency was $110 \mathrm{~Hz}$. The comparisons for the mean flow variables and root mean square axial and normal velocity components were in good agreement with the experiments. In spite the fact that the recirculation zone length was well captured, the rms velocity distributions were underpredicted in this zone. Manickam et al. (2012) presented a detailed discussion on the influence of grid refinement and three subgrid scale models on the results and concluded that a coarse grid is too dissipative. Contrary to what might be expected, the grid refinement has a weak influence on the computation of the St, showing that at least the large scale structures were captured by a course grid LES simulation. The St dependence on the grid was also not observed for the reactive case, independently of the reaction model. The three sub-grid scale models tested were the Smagorinsky (SM), the dynamic Smagorinsky (DS) and the sub-grid scale kinetic energy (KSGS). They showed that the performances of the three different models are more or less equivalent.

Flame Surface Density (FSD) models rely on geometric parameters of the flame front to evaluate its progress. In this case, the laminar flamelet model used in RANS has been adapted to LES by considering a locally laminar flame wrinkled by turbulence. The amount of wrinkling $(\Sigma)$ is measured by the flame surface area per unit volume (Boger and Veynante, 2000). Transport equations for the progress variable and for the wrinkling variable are solved to describe the evolution of the flame since the wrinkling increases the burning rate.

Similar to the $\Sigma$ variable, Weller (1993) proposed a model based on the density of wrinkling $(\Xi)$, which is the flame area per unit area resolved in the mean direction of propagation. Weller (1993) originally developed this model for RANS and later Tabor and Weller (2004) adapted the model for LES. The advantage of using $\Xi$ is that it should be easier to model the transport terms as discussed in Weller (1993), Weller et al. (1998) and Tabor and Weller (2004). This model is used in the present study and will be discussed in detail on the following sections.

In the present study, the SM and dynamic sub-grid models for turbulence were used. For the combustion model, the flame surface wrinkling $(\Xi)$ formulation, developed by Tabor and Weller (2004), was used. The objective of the study was to investigate the performance of a turbulent combustion model when applied to the simulation of pre-mixed turbulent flames behind a triangular obstacle. The review of the literature shows that such type of performance investigation has been conducted previously, with results compared to the Volvo experiment, which has a $\mathrm{Da}$ in the range of 10 and a $\mathrm{Ka}$ of 4 , corresponding to a thickened, wrinkle flame. The present investigation considered an experiment which has a $D a$ of 4.5 and a $K a$ of 1.3; on the range of thin, wrinkled flame, previous investigations found in the open literature did not consider LES simulations in this combustion regime, and the thinner flame thickness poses a more severe test for the turbulent combustion model. The $R e$ for the simulation was 6,690, lower than the $R e$ on the Volvo experiment, which was 204,000 and thus have a significant different flow dynamics. The chosen reactive LES model was evaluated by comparing results with experimental ones obtained by Sanquer (1998), which have not been analyzed before with other LES numerical models.

\section{PROBLEM FORMULATION}

In order to account for turbulence and combustion, the reactive flow governing equations are filtered using the LES concept, and the combustion process is accounted for by following the flame front. Therefore, it is necessary to define the filtering and a variable to account for the regions of burned and unburned gases. In this section, the formulation derived by Weller (1993) and Tabor and Weller (2004) is presented.

\section{PRELIMINARY DEFINITIONS}

In premixed flames, a reaction wave propagates from the burned to fresh gases. The progress variable $c$, that identifies 
this propagation, varies between 0 for fresh gas and 1 for burned gas. The transitions between these values describe the flame front. A progress variable $c$ can be defined based on the normalized temperature $(T)$ or on the reactant mass fraction $(Y)$. Using the temperature, it results in:

$c=\frac{T-T_{u}}{T_{b}-T_{u}}$

Where $b$ subscript stands for burned gas and $u$ subscript, for fresh unburned gas. The flame front propagation is modelled by solving a transport equation for the density-weighted mean reaction regress variable denoted by $b$, where $b=1-c$.

In LES, it is assumed that the dependent variables can be divided into grid scale (GS) and sub-grid scale (SGS) components, such that, for any given dependent variable, it results in:

$\psi=\bar{\psi}+\psi^{\prime}$

Where

$\bar{\psi}=G^{*} \psi \int_{D} G\left(x^{\prime}, \Delta\right) \psi\left(x^{\prime}, t\right) d^{3} x^{\prime}$

Here, $D$ is the computational domain with boundaries $\partial D$, $t$ is the time and $x$, the coordinate directions. The kernel $G=G(x, \Delta)$ is any function of $x$ and of the filter width $\Delta$. G has the properties $\int_{\mathrm{D}} G(x) d^{3} x=1, \lim _{\Delta \rightarrow 0} G(x, \Delta)=\delta(x)$

Introducing a conditional filter (Tabor and Weller, 2004), with an indicator function $l$, results in:

$l(x, t)= \begin{cases}1 & \text { if }(x, t) \\ 0 & \end{cases}$

is in the unburned gas region otherwise.

For a tensor $\psi$ of any rank, one may define $\overline{\bar{\psi}}$ the phaseweighted value of $\psi$ at any point.

$\overline{\bar{\psi}}=G^{\star}(l \psi)=\int_{D} G\left(x-x^{\prime}\right) l\left(x^{\prime}, t\right) \psi\left(x^{\prime}, t\right) d^{3} x^{\prime}$.

Introducing the combustion progress variable $\bar{b}$ as a GS indicator function, we can obtain: $\overline{\bar{\psi}}=\bar{b} \bar{\psi}_{u}$,

where $\bar{b}(x, t)$ is the probability of the point $(x, t)$ being in the unburned gas.

$\bar{b}=\int_{D} G\left(x-x^{\prime}\right) l\left(x^{\prime}, t\right) d^{3} x^{\prime}$

In compressible flow, there is density $(\rho)$ variation, and the product $\overline{\overline{\rho \psi}}$ can be written:

$\overline{\overline{\rho \psi}}=\bar{b} \overline{\rho \psi}$

Where the subscript $u$ indicates the unburned gases.

Defining a density-weight average , in the unburned phase, we can obtain:

$\overline{\rho \psi_{u}}=\bar{\rho} \widetilde{\psi}_{u}$

From Eq. 8 in Eq. 9, results in:

$\overline{\overline{\rho \psi}}=\bar{b} \bar{\rho} \widetilde{\psi}_{u}$

\section{FILTERED CONTINUITY EQUATION}

The governing equations will be written in a coordinate system placed at the flame surface, such that $n_{\perp}$ and $n_{\|}$are the unity vectors pointing the normal $x_{\perp}$ and parallel $x_{\|}$directions to the flame surface. The metrics of this coordinate system are $h_{\perp}$ and $h_{\|}$. This coordinate system is used in order to include the conditional filter based on the progress variable $b$.

The filtered continuity equation reads (Tabor and Weller, 2004):

$\frac{\partial \overline{\bar{\rho}}}{\partial t}+\nabla \cdot \overline{\overline{\rho U}}=\overbrace{\rho\left(U-U_{\mathrm{I}}\right) \cdot n_{\perp}} \Sigma$.

Where $U_{\mathrm{I}}=U+v_{a} n_{\perp}, U_{I}$ is the full velocity on an interface consisting of the movement due to advection term $U$ and the advance of the interface relative to the flow $\boldsymbol{v}_{a} \boldsymbol{n}_{\perp}$.

In the transformed coordinates for $\left(x_{\perp}, x_{\|}\right)$:

$\Sigma=G_{\perp}\left(x_{\perp}-x_{\perp, I}\right) \iint G_{\|}\left(x_{\|}-x_{\|}^{\prime}\right)|\mathfrak{I}| d^{2} x_{\|}$. 
Where $\Im$ represents the Jacobian of the transformation. $|\mathfrak{s}| d^{2} x_{\|}^{\prime}$ is the area element on the surface interface. $\Sigma$ is interpreted as the amount of interface for the filtered component, the flame surface density.

The surface filtering operation $\smile$ is defined as:

$$
\overbrace{\psi}=\frac{1}{\Sigma} \int_{D} G\left(x-x^{\prime}\right) \psi\left(x^{\prime}\right) \delta\left(\left(x^{\prime}-x\right) \cdot n_{\perp}\right) \frac{1}{h_{\perp}} d^{3} x^{\prime} .
$$

From Eq. 10 in Eq. 11, results in:

$$
\frac{\partial \bar{b} \bar{\rho}_{u}}{\partial t}+\nabla \cdot \bar{b} \bar{\rho}_{u} \widetilde{U}_{u}=-\overbrace{\rho \mathrm{v}_{\mathrm{a}}} \Sigma
$$

This surface filtering operation applied to $n_{\perp}$ results in:

$$
\overbrace{n_{\perp}}^{\sim}=\frac{1}{\Sigma} G_{\perp}\left(x_{\perp}-x_{\perp, I}\right) \iint G_{\|}\left(x_{\|}-x_{\|}^{\prime}\right) \boldsymbol{n}_{\perp}\left(x_{\perp, I}, x_{\|}^{\prime}\right)|\mathfrak{I}| d^{2} \boldsymbol{x}_{\|}^{\prime} .
$$

$\overbrace{n_{\perp}}$ can be related to the GS with the $n_{\mathrm{f}}$ direction of the interface:

$\overbrace{n_{\perp}}^{\sim}=\frac{n_{f}}{\Xi}$

Where $\Xi$ represents the total sub-grid surface area by the smoothed surface area in the $\boldsymbol{n}_{f}$ direction:

$\Xi=\frac{1}{\sim_{\perp}}=\frac{\Sigma}{\left|G_{\perp}\left(x_{\perp}-x_{\perp, I}\right) \iint G_{\|}\left(x_{\|}-x_{\|}^{\prime}\right) \boldsymbol{n}_{\perp}\left(x_{\perp, I}, x_{\|}^{\prime}\right)\right| \Im\left|d^{2} \boldsymbol{x}_{\|}^{\prime}\right|}$.

Or:

$\Xi=\frac{\Sigma}{|\nabla \bar{b}|}$

Where $\overline{|\nabla \bar{b}|}$ represents the area of the GS surface.

Combining the burned and unburned gases into the weighted total density results in:

$\bar{\rho}=\bar{\rho}_{u} \bar{b}+\bar{\rho}_{c}(1-\bar{b})$

Such that $\bar{\rho} \tilde{b}=\bar{\rho}_{u} \bar{b}$.
The final continuity equation reads:

$\frac{\partial \bar{\rho} \tilde{b}}{\partial t}+\nabla \cdot\left(\tilde{b} \bar{\rho} \tilde{U}_{u}\right)=-\bar{\rho}_{u} S_{u} \Xi|\nabla \bar{b}|$,

where $S u$ is the laminar flame speed.

\section{FILTERED MOMENTUM AND ENERGY EQUATIONS}

Conditionally filtering the momentum equation gives:

$\frac{\partial \bar{b} \overline{\rho_{u}} \tilde{U}_{u}}{\partial t}+\nabla \cdot\left(\bar{b} \bar{\rho}_{u} \tilde{U}_{u} \otimes \tilde{U}_{u}\right)=-\nabla \bar{b} \bar{p}_{u}$

$+\nabla \cdot\left\{\bar{b}\left(\overline{S_{u}}-B_{u}\right)\right\}+[\overbrace{(p I-S) \cdot n_{\perp}}-\overbrace{\left(\rho v_{\mathrm{a}} U\right)}] \Sigma \quad$.

Where $p$ is the pressure, $S_{u}$ is the laminar flame speed, $\Sigma$ is the flame surface density, $S=\lambda \nabla \cdot U I+2 \mu D$ is the stress tensor and $\boldsymbol{D}=\frac{1}{2}\left(\nabla U+\nabla U^{T}\right)$ is the symmetric part of the strain tensor. The terms in brackets represent the effect of the interface on the momentum balance.

$\boldsymbol{B}_{u}$ represents the SGS stress tensor.

$B_{u}={\overline{(\rho U \otimes U)_{u}}}_{-}-\bar{\rho}_{u} \widetilde{U}_{u} \otimes \widetilde{U}_{u}$

This term requires modeling.

The filtered energy equation is:

$$
\begin{aligned}
& \frac{\partial\left(\bar{b} \bar{\rho}_{u} \widetilde{e}_{u}\right)}{\partial t}+\nabla \cdot\left(\bar{b} \bar{\rho}_{u}{\widetilde{e_{u}}}_{u} \tilde{U}_{u}\right)= \\
& -\left(\bar{b} \bar{\rho}_{u} \nabla \cdot \tilde{U}_{u}+\bar{b} \bar{\rho}_{u} \pi_{u}\right)+\left(\overline{b S_{u}} \cdot D_{u}+\bar{b} \bar{\rho}_{u} \varepsilon_{u}\right) \\
& +\nabla \cdot \bar{b}\left(\bar{h}_{u}-b_{u}\right)+[\overbrace{\left(\rho e v_{a}\right)}-\overbrace{\boldsymbol{h} \cdot \boldsymbol{n}_{\perp}}] \Sigma \ldots
\end{aligned}
$$

Where

$$
\bar{\rho}_{u} \pi_{u}=\overline{(p \nabla \cdot U)_{u}}+\bar{p}_{u} \nabla \cdot \tilde{U}_{u}
$$

and

$$
\bar{\rho}_{u} \varepsilon_{u}=\overline{(S \cdot D)_{u}}+\bar{S}_{u} \bar{D}_{u}
$$


represent the SGS pressure dilatation $\pi$ and dissipation $\varepsilon_{\text {i }}$ (Tabor and Weller, 2004). The total energy at the interface is presented in brackets.

\section{TURBULENCE MODEL}

In the present study, three turbulence models available in OpenFoam were used. These turbulence models are described by Fureby et al. (1997). First, the Smagorinsky classical model is presented:

$\tau_{i j}-\frac{\delta_{i j}}{3} \tau_{k k}=-v_{t} \bar{\rho}\left(\frac{\partial \tilde{u}_{i}}{\partial x_{j}}+\frac{\partial \tilde{u}_{j}}{\partial x_{i}}-\frac{2}{3} \delta_{i j} \frac{\partial \tilde{u}_{k}}{\partial x_{k}}\right)$

$=-2 \bar{\rho} v_{s g s}\left(\widetilde{S}_{i j}-\frac{\delta_{i j}}{3} \widetilde{S}_{k k}\right)$

Where $v_{s g s}$ is the sub-grid viscocity and $\tilde{S}_{i j}=\frac{1}{2}\left(\frac{\partial \tilde{u}_{i}}{\partial x_{j}}+\frac{\partial \tilde{u}_{j}}{\partial x_{i}}\right)$
is the strain tensor.

$v_{s g s}=\left(C_{s} \Delta\right)^{2}|\widetilde{S}|$

The constant $C_{\mathrm{s}}$ is equal to 0.18 .

The second turbulence model is the one-equation model with sub-grid kinetic energy $\tilde{k}$ proposed by Yoshizawa and Horiuti (1985) and given by:

$$
\tilde{k}=\frac{1}{2} \bar{\rho} \tau_{k k}=\frac{1}{2}\left(\left\langle u_{k} u_{k}\right\rangle-\widetilde{u}_{k} \tilde{u}_{k}\right) \quad .
$$

where the operation $\langle a\rangle$ stands for $\widetilde{a}$ in the product $u_{k} u_{k}$ above.

The sub-filter stress is:

$\tau_{i j}=-2 \bar{\rho} C_{v} \Delta \sqrt{k}\left(\tilde{S}_{i j}-\frac{\delta_{i j}}{3} \widetilde{S}_{k k}\right)+\frac{2}{3} \bar{\rho} k \delta_{i j} \quad$,

and turbulent viscosity is: $v_{s g s}=\bar{\rho} C_{k} \Delta \sqrt{k}$.

To close the system of equations, the transport equation for the kinetic energy is used.

$\frac{\partial k}{\partial t}+\frac{\partial \bar{\rho} \tilde{u}_{i} k}{\partial u_{i}}-\frac{\partial v_{k} \nabla k}{\partial u_{i}}=-\bar{\rho} D: B-\varepsilon$ where

$\varepsilon=\frac{C_{e} \bar{\rho} k^{3 / 2}}{\Delta}$

and

$B=\frac{2}{3} k I-2 v_{k} \operatorname{dev}(D) \quad$.

The third turbulence model is the dynamic one-equation model that used the Germano identity, following Ghosal et al. 1995, considering a scaling law and homogeneous flow. The kinetic energy equation is:

$\frac{\partial k}{\partial t}+\frac{\partial \bar{\rho} \tilde{u}_{i} k}{\partial u_{i}}-\frac{\partial v_{k} \nabla k}{\partial u_{i}}=-\bar{\rho} B L-\varepsilon$

where $L$ is the Germano identity (Ghosal et al., 1995):

$L=T-\bar{B}$ with:

$L=(\overline{\bar{u} \otimes \bar{u}}-\overline{\bar{u}} \otimes \overline{\bar{u}})$,

$T=(\overline{\overline{u \otimes u}}-\overline{\bar{u}} \otimes \overline{\bar{u}})$,

$\bar{B}=(\overline{\overline{u \otimes u}-\bar{u} \otimes \bar{u}})$.

\section{SUBGRID COMBUSTION MODEL}

Models for the SGS stress tensor, flux vectors, dissipation and filtered reaction rates are used to close the governing equations. The models for the SGS stress tensor and flux vectors are the standard ones used in LES, since they do not depend on reacting flow data.

A flamelet model with conditional filtering for LES is used to derive the transport equations. This model considers conditions of the Klimov-Williams criterion $(K a=1)$ in the Borghi diagram (Borghi and Destriau, 1998). Instead of using the flame propagation speed in terms of the laminar flame area per unit volume $\Sigma$ and the degree of wrinkling of the flame at a point in the domain, the present model (Weller, 1993) uses the flame surface density and a wrinkling surface function $\Xi$. The function $\Xi$ is the average flame area per unit volume divided by the projected area in the mean direction of propagation.

In the flamelet regime, turbulent motions are slow and do not affect the flame structure. The disturbance velocity $u^{\prime}$ considers how a rotation speed of the larger turbulent motion wrinkles the flame surface front (Veynante, 2006). In the reaction zone, the characteristic scales for the reaction processes are below the filter, so that, in reacting LES, a proper treatment (modeling) 
of the reaction zone is needed. In other words, equations for the geometric variables $\bar{b}$ and $\Xi$ are needed.

\section{Sub-grid scale model for $\bar{b}$}

In Eq. 20, the right hand side needs a SGS model, which is based on the conditional filter of unburned gas velocity $\widetilde{U}_{u}$. This term is modeled using:

$\widetilde{U}_{u}=\widetilde{U}+(1-\tilde{b}) \widetilde{U}_{u c}$

where $\widetilde{U}_{u c}$ is the slip velocity of the unburned minus burned gas $\tilde{U}_{u c}=\widetilde{U}_{u}-\widetilde{U}_{c}$.

This is similar to the properties of laminar flame for LES:

$\widetilde{U}_{u c}=\left(\frac{\bar{\rho}_{u}}{\bar{\rho}_{c}}-1\right) S_{u} \Xi n_{f}-\mathcal{D} \frac{\nabla \tilde{b}}{\widetilde{b}(1-\tilde{b})}$,

where $\mathcal{D}$ is the diffusion coefficient of the sub-grid, and $n_{f}=\nabla b /|\nabla b|$ is the flame normal direction.

From Eq. 20, one arrives at the final equation for $\widetilde{b}$ (Tabor and Weller, 2004):

$\frac{\partial \bar{\rho} \tilde{b}}{\partial t}+\nabla \cdot(\bar{\rho} \widetilde{U} \widetilde{b})-\nabla \cdot(\bar{\rho} \partial \nabla \widetilde{b})=-\overline{\rho_{u}} S_{u} \Xi|\nabla \bar{b}|$.

\section{Sub-grid scale modeled equation for $\Xi$}

From the transport equation for the sub-grid flame area density $\Sigma$, proposed by Weller (1993), an equation for $\Xi$ is obtained from the relation $\Xi=\Sigma /|\nabla \bar{b}|$ and the resolved unburned gas volume fraction $b$ (Tabor and Weller, 2004):

$$
\begin{aligned}
& \frac{\partial \Xi}{\partial t}+\widetilde{U} \cdot \nabla \Xi=-\Xi \overbrace{n_{\perp} \cdot \nabla U_{I} \cdot n_{\perp}}+\Xi n_{f} \cdot \nabla \widetilde{U_{t}} \cdot n_{f} \\
& +\left(\widetilde{U_{t}}-\widetilde{U_{I}}\right) \cdot \frac{\nabla|\nabla \bar{b}|}{|\nabla \bar{b}|} \Xi .
\end{aligned}
$$

where $\widetilde{U}_{t}$ is the surface-filtered effective velocity of the fla- me and $\widetilde{U}_{I}$ is the local instantaneous velocity of flame surface.

The proposed model for Eq. 38 considers wrinkling generation $G \Xi$. and removal $2(\Xi-1)+$, such that (Tabor and Weller, 2004): $\frac{\partial \Xi}{\partial t}+\widetilde{U}_{s} \cdot \nabla \Xi=G \Xi-R(\Xi-1)+\max \left[\left(\sigma_{s}-\sigma_{t}\right), 0\right] \Xi$,

$\sigma_{t}=\frac{1}{2}\left\|\nabla \widehat{U}_{t}+\nabla \widehat{U}_{t}^{T}\right\|$,

and

$\sigma_{s}=\frac{1}{2}\|\overrightarrow{\nabla \hat{\mathrm{U}}_{\mathrm{I}}}+\nabla \overbrace{\mathrm{U}_{\mathrm{I}}^{\mathrm{T}}}^{\mathrm{T}}\|$.

The models for $G$ and $R$, which accounts for the interaction between turbulence and flame, are:

$G=R \frac{\Xi_{e q}-1}{\Xi_{e q}}$,

and

$R=\frac{0.28}{\tau_{\eta}} \frac{\Xi_{e q}^{*}-1}{\Xi_{e q}^{*}}$,

where

$\Xi_{e q}^{*}=1+0.62 \sqrt{\frac{u^{\prime}}{S_{u}}} R e_{\eta}$,

$\Xi_{e q}=1+2(1-\bar{b})\left(\Xi_{e q}^{*}-1\right)$.

where $\tau_{\eta}$ is the Kolmogorov time, $u^{\prime}$ is the intensity of turbulence in the sub-grid and $R e_{\eta}$, is the Kolmogorov Reynolds number.

Finally, an equation for the laminar flame speed (Tabor and Weller, 2004) is needed. A proposed transport equation is:

$\frac{\partial S_{u}}{\partial t}+\overbrace{U_{s}}^{\infty} \cdot \nabla S_{u}=-\sigma_{s} S_{u}+\sigma_{s} S_{u}^{\infty} \frac{\left(S_{u}^{0}-S_{u}\right)}{\left(S_{u}^{0}-S_{u}^{\infty}\right)} \quad$.

The convective velocity of the laminar flame is the filtered surface speed $\overbrace{U_{s}}$. And the superscripts 0 and $\infty$ stand for the value of unstrained flame speed and the value at equilibrium, respectively. 


\section{NUMERICAL MODEL}

The present investigation uses OpenFoam, an open source $\mathrm{C}++$ collection of libraries for transport equations. The model equations are solved numerically based on a cell centered unstructured finite volume scheme. The solution is based on a segregated approach. For time integration, a first order explicit Euler method is used. For spatial discretization, second and third order Total Variation Diminishing (TVD) schemes are used. The PISO algorithm (pressure-implicit split-operator) is used to solve the pressure-velocity coupling. The discretization method is the standard Gauss finite volume integration. The combustion solver called Xifoam, available on OpenFoam, was used. This premixed turbulent combustion model is described in the previous section - Preliminary definitions (Weller et al., 1998; Tabor and Weller, 2004).

Both reactive and non-reactive cases were run on a SGI Altix XE 1300 cluster with Intel x86 64 processors, running SUSE Linux Enterprise Server version 10. The cluster has 144 cores with $432 \mathrm{~GB}$, DDR3, $1066 \mathrm{MHz}$ RAM. For the non-reactive cases running with 64 cores, the average seconds per iteration computational time for a simulation using SM was 1.47 seconds. The clock time was about $12 \mathrm{~h} 52 \mathrm{~m}$. For the reactive case running with 136 cores, the average seconds per iteration for a simulation using the dynamic model was 8.04 seconds.

\section{INITIAL AND BOUNDARY CONDITIONS}

The geometry of the channel with a bluff body flame holder is presented in Fig. 1. It consists of a channel $0.600 \mathrm{~m}$ long, $0.160 \mathrm{~m}$ wide and $0.0288 \mathrm{~m}$ tall. The Re based on the inlet velocity and twice the channel height $\left(R e=U_{\text {axe }} 2 h / v\right)$ is 6,690 . The obstacle used as flame holder is an equilateral triangular cross section obstacle whose backside is located $0.160 \mathrm{~m}$ from the entrance. The obstacle blockage is $33 \%$ of the total area and corresponds to the $r-65$ test case in Sanquer's experiment (Sanquer, 1998).

First, only inert simulations are considered with an initial temperature of $300 \mathrm{~K}$. At the outflow boundary, a pressure wave transmissivity boundary condition is used for pressure (Candel, 1992). A uniform velocity profile is imposed at the entrance with a speed of $U_{\text {axe }}=3.1 \mathrm{~m} / \mathrm{s}$ to match the experimental value. On top of the uniform average velocity profile at the inlet, a fluctuation is added using a routine available on OpenFoam that mimics the turbulent statistical properties. This procedure is necessary since the inlet turbulence determines the flow behavior on the domain, as discussed by Tabor and Baba-Ahmadi (2010).

The flow is considered periodic in the spanwise direction. A wall function is used in the channel walls, as shown schematically in Fig. 2 (Jayatilleke, 1969).

For reacting flows, propane $\left(\mathrm{C}_{3} \mathrm{H}_{8}\right)$ premixed with air is considered. The mixture is ignited behind the obstacle in the recirculation zone to achieve a proper performance and avoid flame blow off. The ignition point is located at $0.05 \mathrm{~m}$ behind the obstacle in the center of the recirculation zone. A combustion time of $3 \mathrm{~ms}$ was used before collecting data to avoid numerical transients and allow time to achieve stable combustion behavior. The imposed initial flame speed was $0.256 \mathrm{~m} / \mathrm{s}$ and the initial condition for the regress variable was $b=1$.

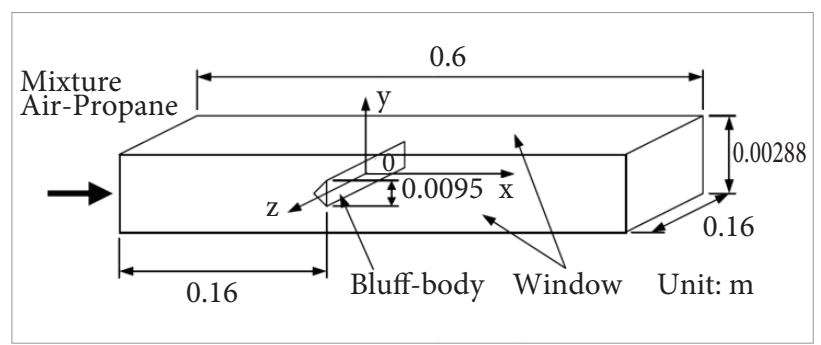

Figure 1. Scheme of Sanquer's experiment.

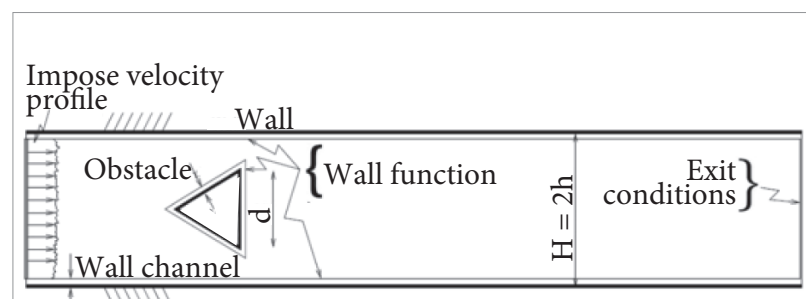

Figure 2. Schematic boundary conditions. Turbulent velocity profile at the channel entrance, walls and obstacle.

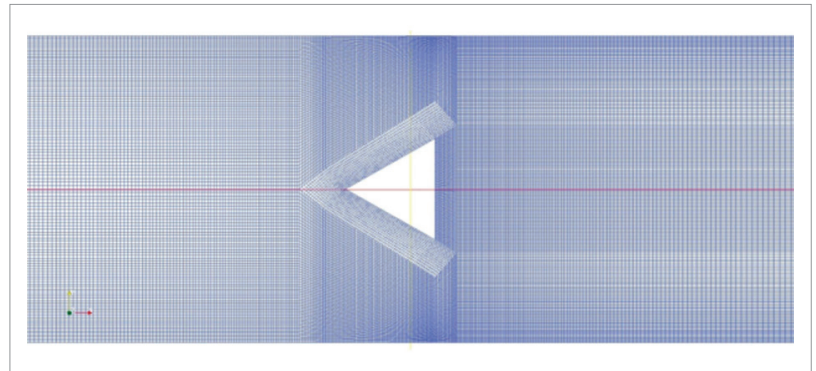

Figure 3. Grid structure around the obstacle. 
The grid used in the present study is composed of 388,355 volumes for two-dimensional simulations and 2,300,000 volumes for three-dimensional simulations. Figure 3 shows the grid topology around the obstacle. Lower grid densities were tested but it was not possible to recover the recirculation zone size obtained by Sanquer (1998). The adequacy of the grid was also tested through the turbulent decay rate, which should follow the Kolmogorov $-5 / 3$ decay, as discussed in the next section.

A detailed study of grid requirement using grid quality assessment techniques was presented by Manickam et al. (2012). They simulated the Volvo experiment using LES and a flame surface wrinkling reaction model based on the progress variable, similar to the model used in this investigation. Their finest grid size has 2.4 million cells, close to the grid size used in the present investigation. Their intermediate and fine grids were able to recover all relevant experimental results for mean and turbulent quantities as well as the St. Nevertheless, even the fine grid presented lower quality near the flamelet region. Considering that the Volvo experiment has a $R e$ much larger than the Sanquer's experiment, it is safe to say that the fine grid for the Volvo higher Re should capture all the relevant structures for the Sanquer's lower Re experiment and will allow the necessary level of accuracy for the sub-grid scale model.

The instantaneous velocity was monitored in order to establish the time for which the flow can be considered periodic stationary. Figure 4 shows the history of longitudinal velocity. As can be seen, the transition lasts 0.18 seconds, so the flow can be considered periodic stationary beyond that.

\section{RESULTS}

In this section, the performance of the turbulent combustion models presented in problem formulation section and available on OpenFoam is assessed for the flow conditions considered by Sanquer (1998).

First, results are presented in terms of frequencies $f_{\mathrm{q}}$ and Strouhal numbers $\left(S t=f_{\mathrm{q}} d / U_{\text {axe }}\right)$ of the vortex emission frequency behind the obstacle, where $d$ is the obstacle height and $U_{\text {axe }}$ is the maxim velocity at the channel entrance. The size of the average recirculation zone $X_{\mathrm{r}}$ is also compared. Next, the energy spectrum is analysed in order to verify the adequacy of the computational grid and to compare the inertial range against the $-5 / 3$ turbulence decay rate for both inert and reactive cases (Pope, 2000). Then, streamwise and normal velocity profiles are compared to the experimental results in the recirculation zone and downstream of the recirculation zone. Finally, the progress variable and temperature profiles are presented.

The recirculation zone and the energy spectra are determined through the velocity data after the simulation reaches the stationary periodic state. Once the length of the recirculation zone $X_{\mathrm{r}}$ is determined, one can calculate the $S t$ to compare it with experimental results. To determine the values of the energy spectrum, the Fast Fourier Transform (FFT) of the oscillatory instantaneous velocity is taken. This information is used to determine if the simulation captures the large turbulent scales and models the sub-grid scales correctly.

\section{INERT FLOW CASE}

First, inert flow results are presented. Table 1 presents the frequencies, $S t$ and recirculation zone length for the present

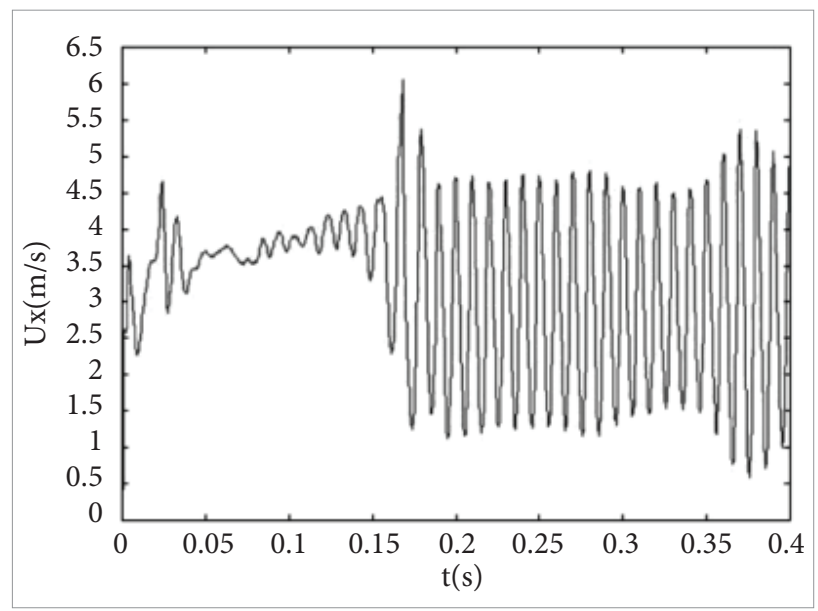

Figure 4. Evolution of velocity component $U x$.

Table 1. Comparison of numerical Large Eddy Simulation (LES) and Reynolds Averaged Navier Stokes (RANS) results with experimental results.

\begin{tabular}{|l|c|c|c|c|}
\hline & $f q[\mathrm{~Hz}]$ & $f q d / U$ & $\Delta X_{r}[\mathrm{~m}]$ & $X_{r} / d$ \\
\hline $\begin{array}{l}\text { Experimental - } \\
\text { Sanquer (1998) }\end{array}$ & 89 & 0.276 & 0.0204 & 2.12 \\
\hline $\begin{array}{l}\text { LES (current work) } \\
\text { RANS - Dourado }\end{array}$ & 93.5 & 0.284 & 0.023 & 2.42 \\
\hline$(2003)$ & 87 & 0.2694 & 0.0222 & 2.31 \\
\hline
\end{tabular}


LES simulation and for RANS simulations performed by Dourado (2003). The results show good agreement with those obtained experimentally.

The length of the recirculation zone based on the evolution of the average velocity $U x$ was determined taking the average values along the $X$ axis downstream of the obstacle. Figure 5 shows the profile of the average velocity used to define the recirculation zone as shown schematically in Fig. 6. The length of the recirculation zone found in the present simulation is $0.023 \mathrm{~m}$, while the length found by Sanquer (1998) is $0.0204 \mathrm{~m}$, and a simulation based on a RANS model (Dourado, 2003) gives $0.0222 \mathrm{~m}$. The recirculation zone length differs approximately $11.5 \%$ from the experimental value.

To determine the vortex emission frequency, a temporal Fourier analysis was used (Dourado, 2003). For the numerical simulation using LES, a value of $93.5 \mathrm{~Hz}$ was found, as shown in Fig. 7, while the experimental result was $f_{\mathrm{q}}=89 \mathrm{~Hz}$. These values correspond to a $S t=0.284$ for the simulation and $S t=0.276$ from the experiment with a difference of only $3 \%$. The RANS model gives $f q=87 \mathrm{~Hz}$ and $S t=0.2694$, with $2.39 \%$ difference in the $S t$ to the experimental result in this inert case.

For the three-dimensional simulations, the energy spectrum shown in Figs. 8 through 11 has the $-5 / 3$ energy decay rate expected for large $R e$ turbulent flows. Figures 8 and 9 are the spectrum for the streamwise velocity component at $X / X_{\mathrm{r}}=1.4$, in two different distances from the channel centerline, $y / h=0.1$ and $y / h=0.41$, respectively. Figures 10 and 11 are spectrum at those two same positions, but for the normal velocity component. On the energy spectrum in Figs. 9 and 11, peaks at about $80 \mathrm{~Hz}$ can be identified. These peaks correspond to the large scale vortex shedding frequency measured above the channel centerline. At the centerline on Figs. 8 and 10, the peaks seem to be at $100 \mathrm{~Hz}$ but the spectrum is not conclusive.

Filtered longitudinal $U x$ and normal $U y$ component velocity profiles at $X / X_{\mathrm{r}}=0.8$ and $X / X_{\mathrm{r}}=1.4$ are shown in Figs. 12 to 15 . Figure 12 shows the longitudinal velocity profiles in the normal direction obtained in the simulation at $X / X_{\mathrm{r}}=0.8$. The results are in good agreement with the experimental results, but the dynamic model overpredicts the velocities in the region behind the obstacle and does not show the reverse flow in the recirculation zone. Downstream of the recirculation zone at $X / X_{\mathrm{r}}=1.4$ (Fig. 13), the longitudinal velocity distributions are also close to the experimental results, even for the dynamic model, which now shows a moderate underprediction. The results obtained with the SM and one equation models are more accurate.

The normal velocity component is underpredicted by all models in the regions behind the obstacle $(y / h<0.6$; Fig. 14), with the dynamic model showing the worse results. Approaching the upper wall (y/h greater than 0.6) the turbulence models give better predictions, but the Smagorinsky model gives results a little higher than the experiments. Never the less, the results are within the limits of the experimental error.

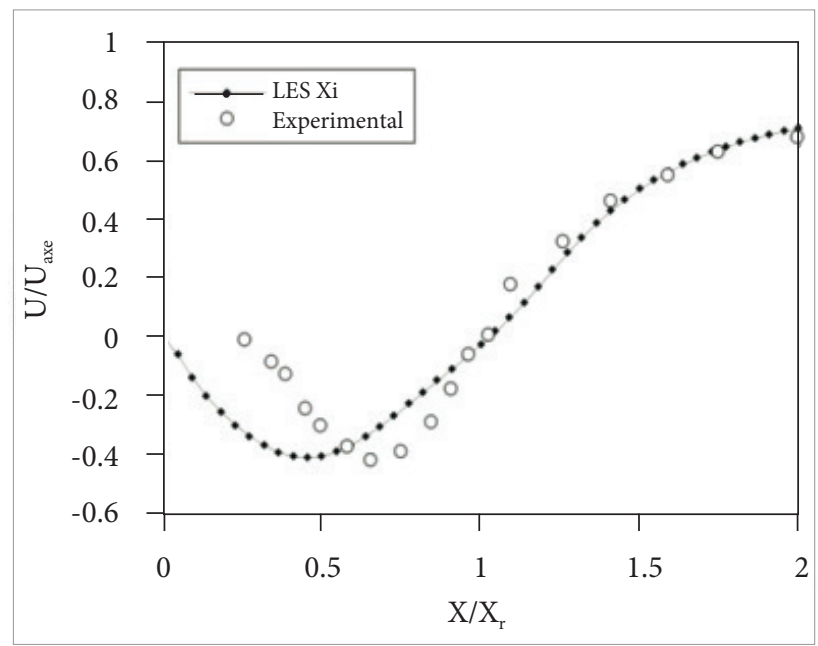

Figure 5. Profile of the average velocity $\bar{u}$ in the recirculation zone.

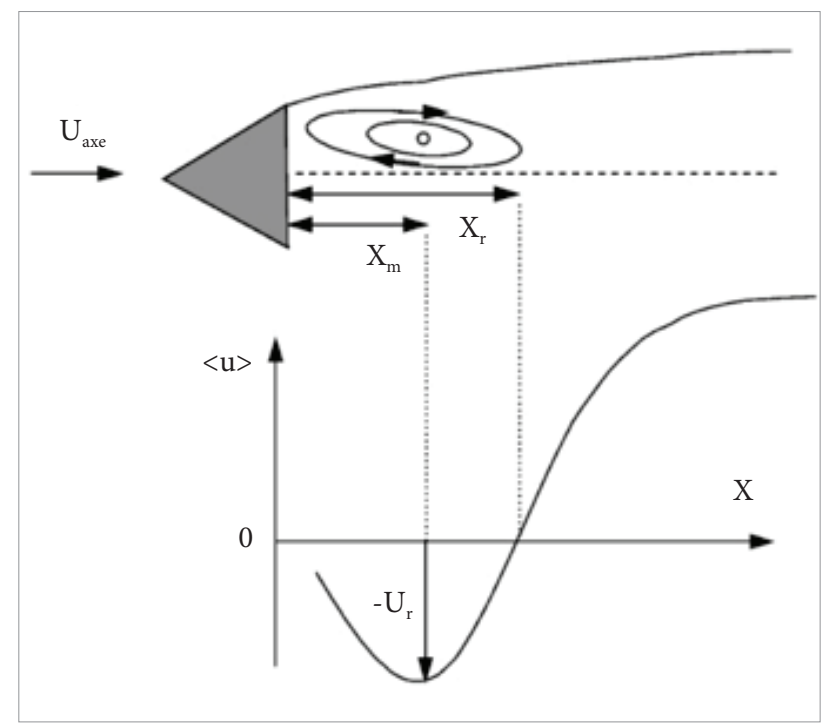

Figure 6. Schematic representation of the velocity distribution (Sanquer, 1998). 
Further downstream at $X / X_{\mathrm{r}}=1.4$ (Fig.15), the turbulence models show a better performance, but the dynamic model captures an upwash velocity profile where the other models and the experimental results show a downwash normal velocity distribution. This error occurs in the region behind the obstacle, but approaching the upper wall, all models show improved results. Further investigations are underway to clarify this dynamic model behavior.

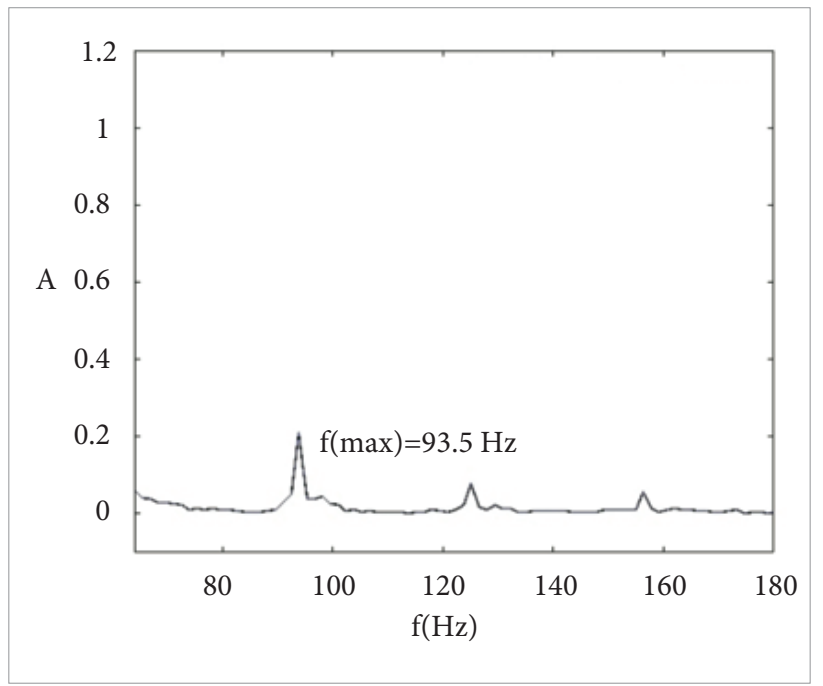

Figure 7. Frequency spectrum identifying the vortex emissions frequency.

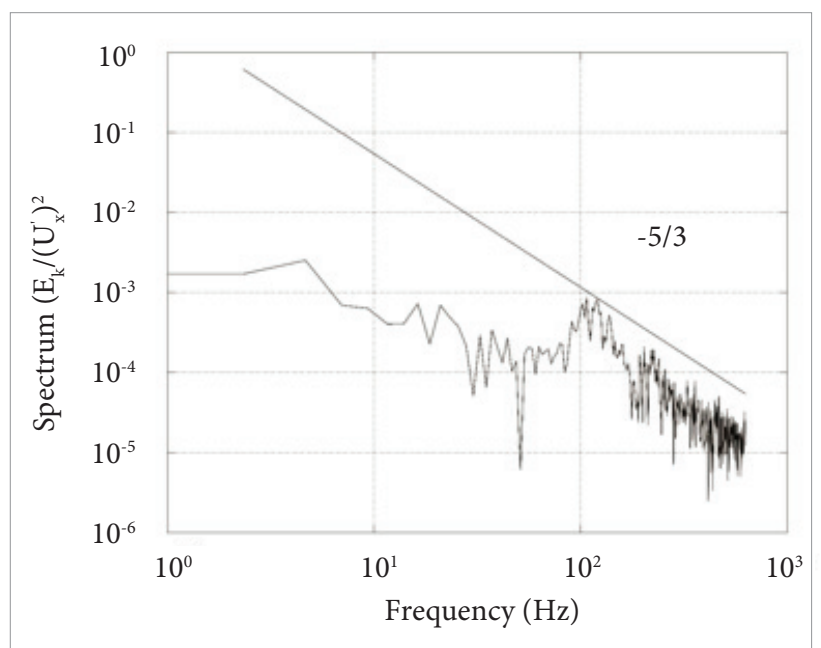

Figure 8. Energy spectrum of the longitudinal velocity components $U_{x}$ at $X / X_{r}=1.4, y / h=0$. Three-dimensional simulation.

\section{REACTIVE CASE}

For the reactive flow, pre-mixed propane $\left(\mathrm{C}_{3} \mathrm{H}_{8}\right)$ and air with an equivalence ratio equal 0.65 is considered and compared to Sanquer (1998) results.

The energy spectrum of the spanwise fluctuation velocity kinetic energy for the reactive case is shown in Fig. 16. The velocity time series was taken at $X / X_{\mathrm{r}}=1$ and $y / h=0$. As in the inert case, the simulation recovers

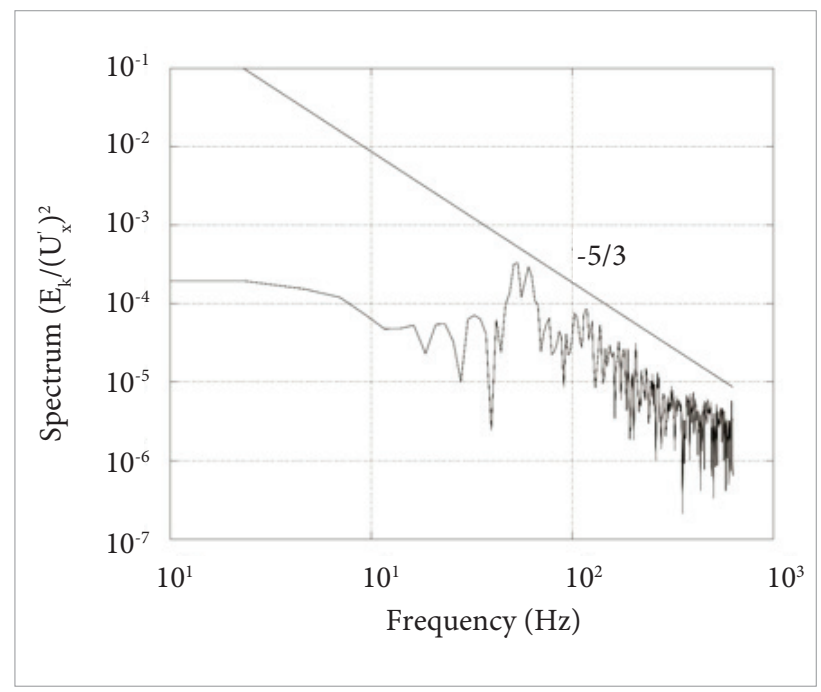

Figure 9. Energy spectrum of the longitudinal velocity components $U_{\mathrm{x}}$ at $X / X_{r}=1.4, y / h=0.41$. Three-dimensional simulation.

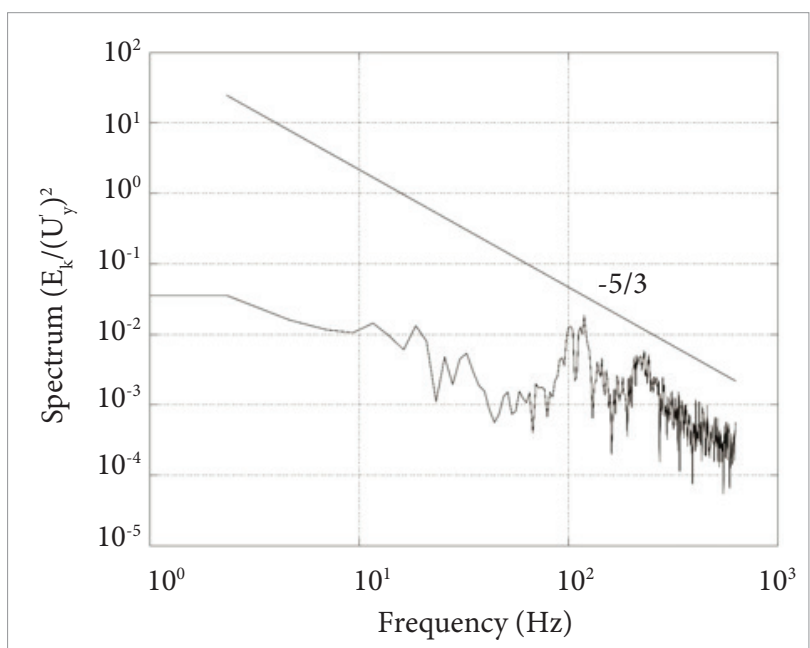

Figure 10. Energy spectrum of the normal velocity components $U_{y}$ at $X / X_{r}=1.4, y / h=0$. Three-dimensional simulation. 


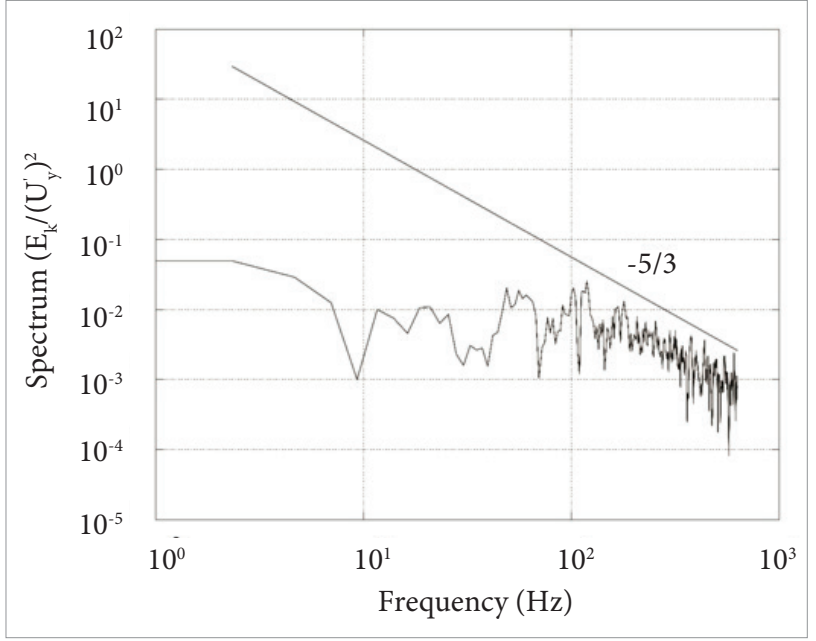

Figure 11. Energy spectrum of the normal velocity components $U_{y}$ at $X / X_{r}=1.4, y / h=0.41$. Three-dimensional simulation.

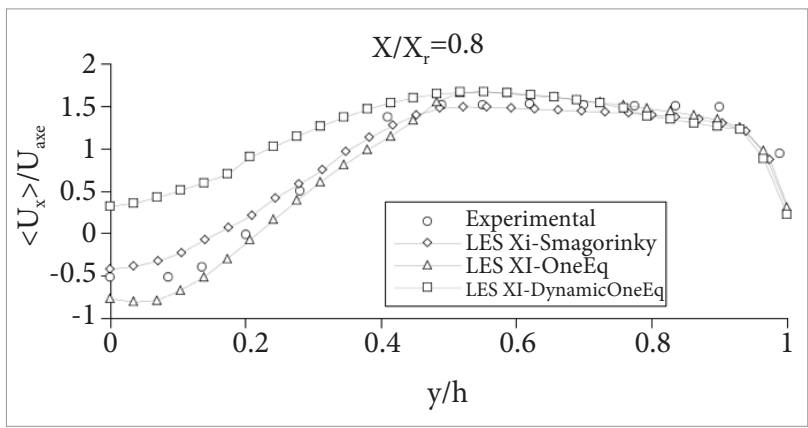

Figure 12. Inert case. One equation, dynamic and Smagorinsky models. Mean longitudinal velocity profile $U_{\mathrm{x}}$ at $X / X_{\mathrm{r}}=0.8$.

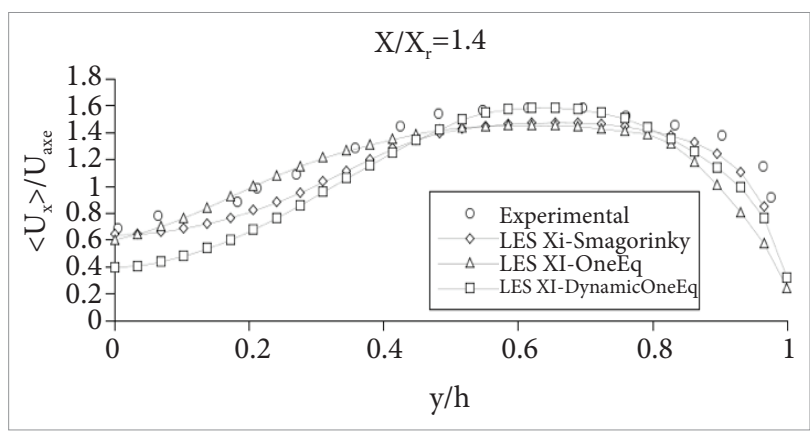

Figure 13. Inert case. One equation, dynamic and Smagorinsky models. Mean longitudinal velocity profile $U_{x}$ at $X / X_{r}=1.4$.

the $-5 / 3$ decay rate. A peak on the energy spectrum can be observed at $94 \mathrm{~Hz}$, which is close to the experimental value of $89 \mathrm{~Hz}$ observed by Sanquer (1998).

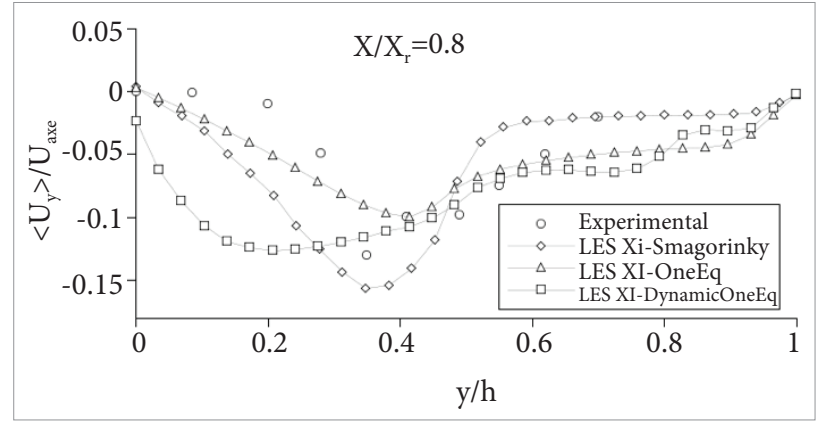

Figure 14. Inert case. One equation, dynamic and Smagorinsky models. Mean normal velocity profile $U_{y}$ at $X / X_{r}=0.8$.

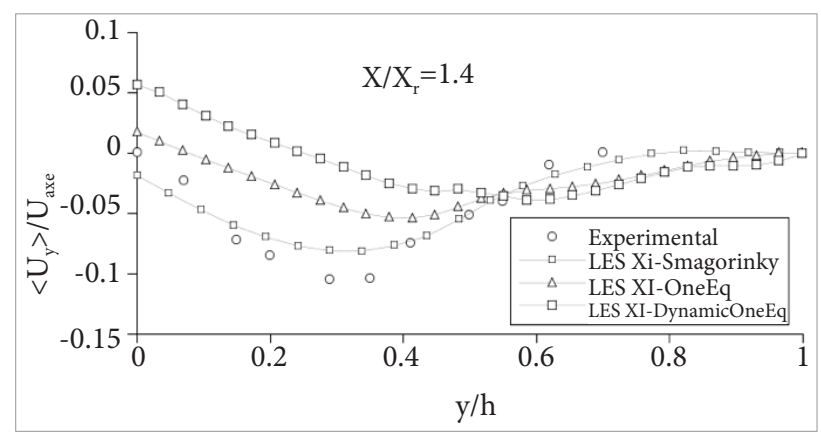

Figure 15. Inert case. One equation, dynamic and Smagorinsky models. Mean normal velocity profile $U_{\mathrm{y}}$ at $X / X_{\mathrm{r}}=1.4$.

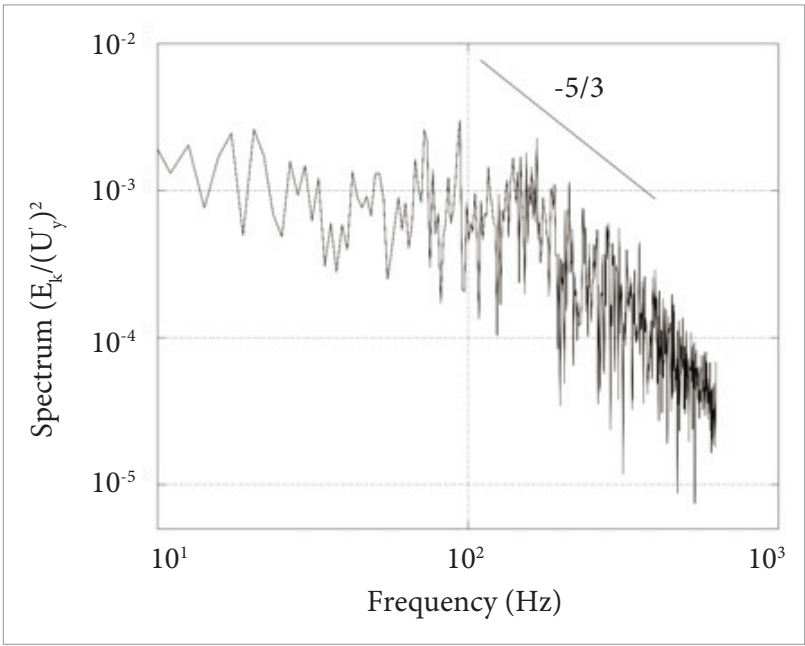

Figure 16. Energy spectrum of the normal velocity component at $X / X_{r}=1$ and $y / h=0$.

Streamwise and normal velocity profiles at two different positions in the streamwise direction $\left(X / X_{\mathrm{r}}=0.8\right.$ and $X / X_{\mathrm{r}}=1.4$ ) are presented in Figs. 17 through 20. Again, 
dynamic, one equation and Smagorinsky SGS model results are presented. The streamwise velocity distribution at $X / X_{\mathrm{r}}=0.8$ is very close to the experimental observed velocity distribution for all the three models, as shown in Fig. 17. At $X / X_{\mathrm{r}}=1.4$, the results for the streamwise velocity also compare very well with the experiments, as shown in Fig. 18, but the one equation model overpredicts the velocity distribution.

The values of the normal velocity component at $X / X_{\mathrm{r}}=0.8$ are presented in Fig. 19. The one equation and the Smagorinsky models are in good agreement with the experimental results in the region behind the obstacle $(y / h<0.4)$, while the dynamic model underpredicts the experimental results. Away from the central region, approaching the upper wall, the trend is reversed and the dynamic model shows better results than the Smagorinsky and one equation models. These results are not conclusive to which turbulence model is more suitable for the reactive case simulation. This same behavior is also observed for the normal velocity component at $X / X_{\mathrm{r}}=1.4$ (Fig. 20), with different turbulent models performing better or worse at different regions of the flow domain, but all models capturing the general trends of the experimentally observed velocity distributions.

The progress variable computed by the dynamic model is presented in Figs. 21 and 22 and compared to the experimental distribution. The simulation captures the general behavior of the progress variable quite well. Despite the fact that the simulation overpredicts the progress variable inside the flame zone, the flame front at $c=0.05$ around $y / h=0.6$ matches the experimental value in $X / X_{\mathrm{r}}=0.35$ and underpredicts for $X /$ $X_{\mathrm{r}}=1.4$. Also, Sanquer (1998) states that the experimental results seem to be displaced to the left.

For the 3-D reactive case, a value of $1,750 \mathrm{~K}$ was obtained for the flame average temperature with the dynamic model. The theoretical adiabatic flame temperature for a equivalence ratio of 0.65 corresponds to $1,750 \mathrm{~K}$ for this fuel. Figures 23 and 24 show the temperature distribution in the normal direction with a profile corresponding to a premixed flame.

The vorticity distribution in the inert and reactive cases can be compared with the help of Figs. 25 and 26 . These figures show the flow structure in the $(x, y)$ cut at the center of the channel. As in Park's discussion (Park and Ko 2011), the chemical reaction has a stabilizing effect on the vortex shedding.

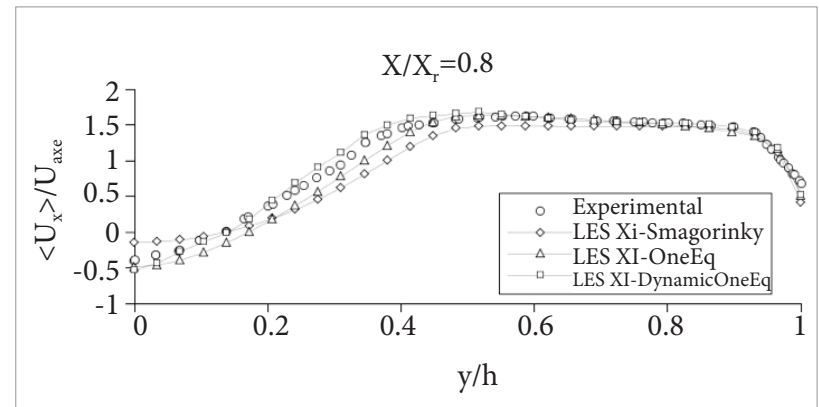

Figure 17. Mean longitudinal velocity profile $U_{\mathrm{x}}$ at $X / X_{\mathrm{r}}=0.8$. Reactive case.

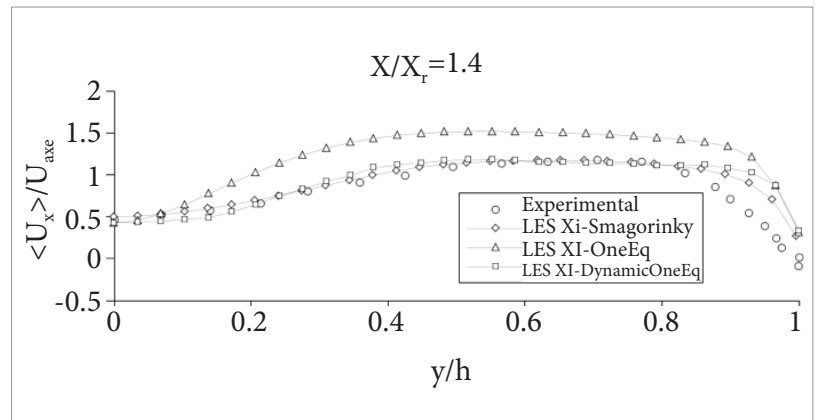

Figure 18. Mean longitudinal velocity profile $U_{x}$ at $X / X_{r}=1.4$. Reactive case.

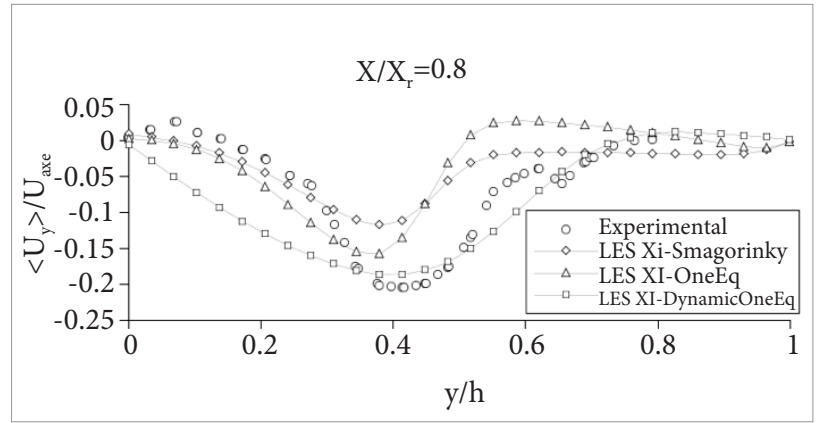

Figure 19. Mean normal velocity profile $U_{y}$ at $X / X_{r}=0.8$. Reactive case.

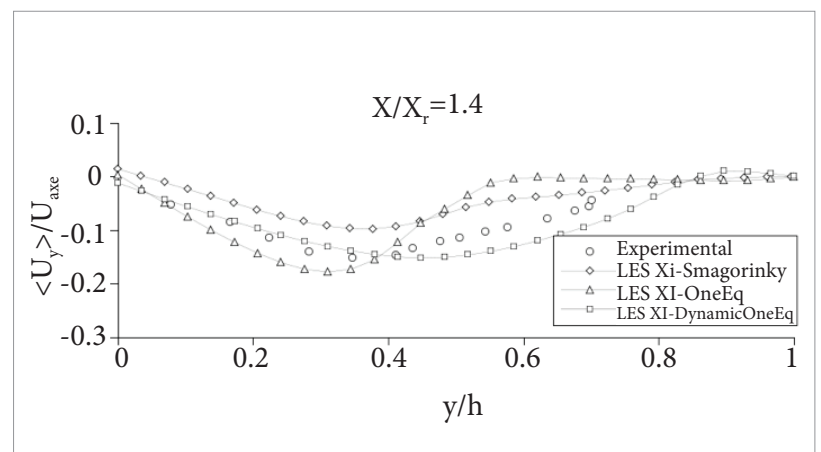

Figure 20. Mean normal velocity profile $U_{y}$ at $X / X_{r}=1.4$. Reactive case. 


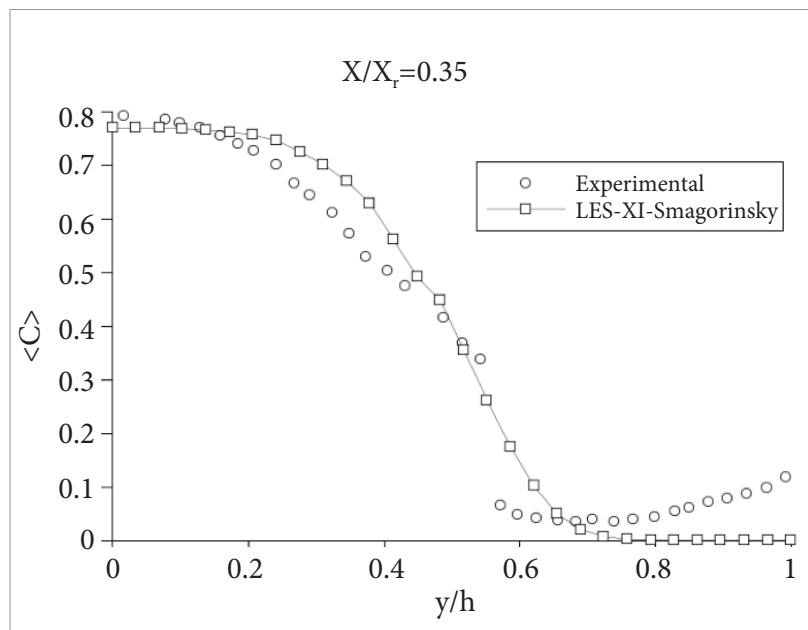

Figure 21. Profile of the progress variable at $X / X_{r}=0.35$.

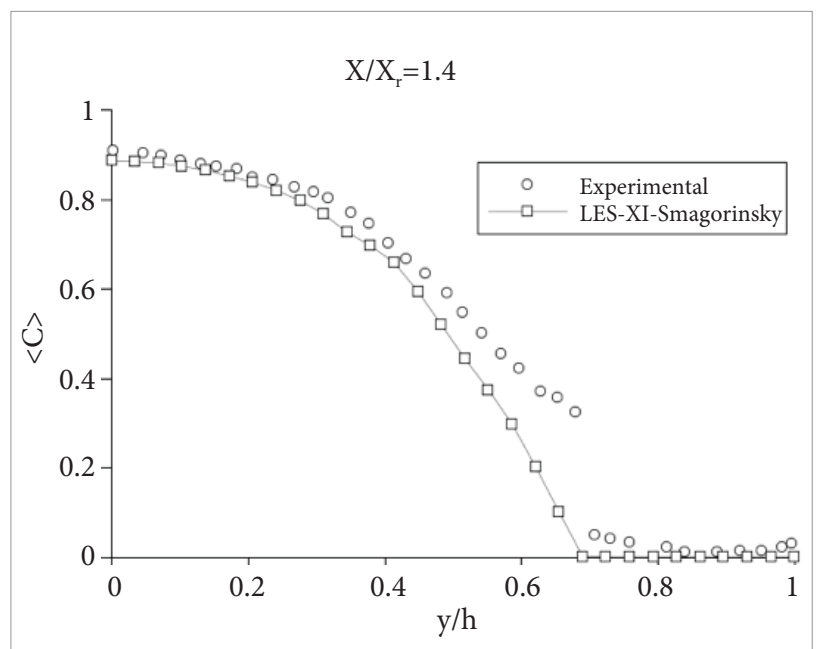

Figure 22. Profile of the progress variable at $X / X_{r}=1.4$.

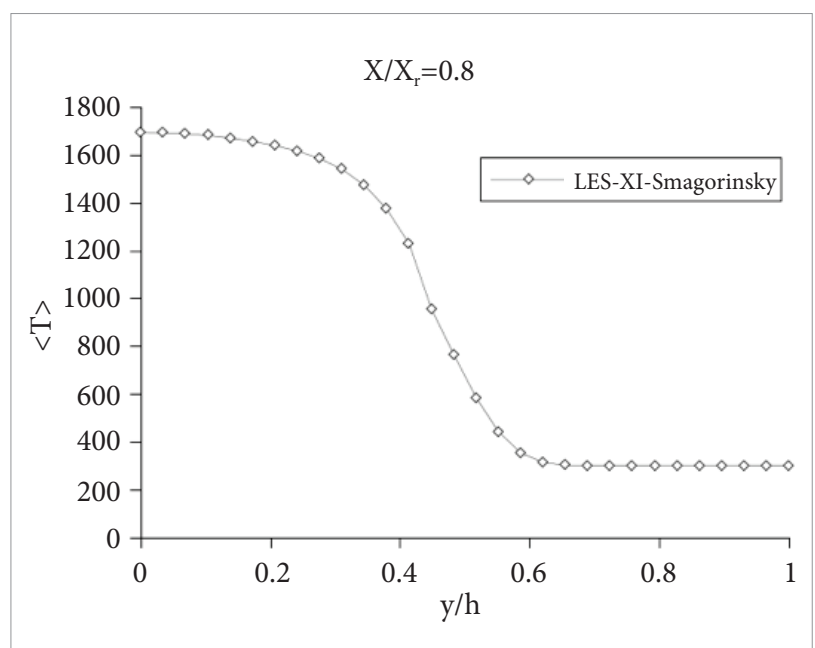

Figure 23. Mean temperature profile at $X / X_{r}=0.8$.

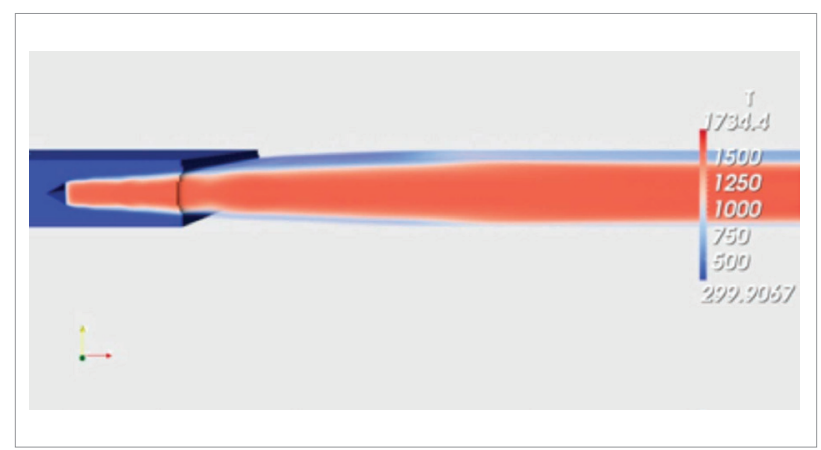

Figure 24. Temperature contours behind the bluff body.

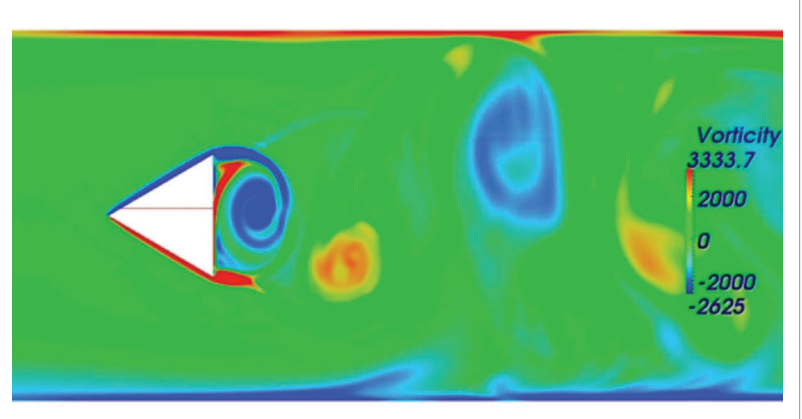

Figure 25. Inert flow spanwise vorticity.

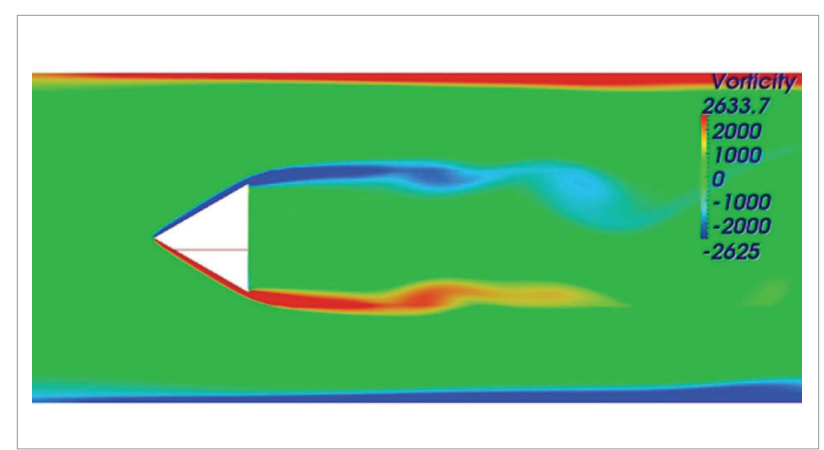

Figure 26. Reactive flow spanwise vorticity.

The inert case clearly shows the Karman vortex street behind the bluff body, while in the reactive case the characteristic alternating vortex structure starts further downstream with a much weaker strength. The vorticity near the bluff body has a stretched topology on the reactive case and the wake spreading is lower than the wake spreading of the inert case, which is in agreement with the behavior described by Park and Ko (2011).

\section{CONCLUSIONS}

Results from numerical simulations of inert and reactive flows were compared to experimental results obtained by Sanquer 
(1998). Smagorinsky, one equation and dynamic LES models were used and the reaction was simulated using the $\Xi$ density of wrinkling model. The experiments are in the thin, wrinkled flame regime with a $R e$ of 6,690, a $D a$ of 4.5 and a $K a$ of 1.3 .

For the inert flow case, the simulations using LES good results were obtained for the $S t$, with an error of the order of $3 \%$. The size of the recirculation bubble is close to the size observed experimentally but the margin of error is larger, around $12 \%$. But these values are not absolute values since the experimental results also have a margin of error. The energy decay on the inertial range was correctly captured at $-5 / 3$. As far as the velocity distribution is concerned, the streamwise velocity component shows good agreement with the experimental values, but the normal velocity components are somewhat off, in part as a consequence of the over-predicted recirculation zone length.

The results for the reactive flow case show similar performance with the inert case. The energy decay rate in the inertial range was correctly captured and the vortex sheading frequency was close to the experimental value. The progress variable and the correct behavior considering the stabilization of the vortex sheading behind the obstacle for the reactive case were captured by the simulation. Again, on the reactive case, the velocity distribution was somewhat off from the experimental measurements. Regarding the velocity profile, it is still interesting to observe that the SM gives a better result than the dynamic model used in these simulations. The results may be improved by tailoring the dynamic turbulent model coefficients. In general, the results for all three models are relatively similar and the differences between these models are small. Therefore, since the dynamic model has a higher computational cost, it may be worthwhile to use the Smagorinsky or the one equation model.

\section{ACKNOWLEDGMENTS}

The authors would like to thank the Instituto de Aeronáutica e Espaço for the use of their computational facilities in the Laboratory of Liquid Propulsion and Brazilian Government CAPES for the financial support.

\section{REFERENCES}

Akula, R.A., Sadiki, A. and Janicka, J., 2006, "Large Eddy Simulation of Bluff Body Stabilized Flame by Using Flame Surface Density Approach". Proceedings of the European Conference on Computational Fluid Dynamics. ECCOMAS CFD.

Bai, X.S. and Fuchs, L., 1994, "Modeling of turbulent reactive flows past a bluff body: Assessment of accuracy and efficiency". Computers \& Fluids, Vol. 23, No. 3, pp. 507-521.

Borghi, R. and Destriau, M., 1998, "Combustion and Flames: chemical and physical principles". Éditions TECHNIP.

Boger, M. and Veynante, D., 2000, "Large eddy simulations of a turbulent premixed V-shape flame". In: Advances in Turbulence VIII, Proceedings of the Eighth European Turbulence Conference, Barcelona, pp. 449-452.

Candel, S., 1992, "Combustion instabilities coupled by pressure waves and their active control". Symposium (International) on Combustion, Vol. 24, No 1, pp. 1277-1296.

Chaudhuri, S., Kostka, S., Tuttle, S.G., Renfro, M.W. and Cetegen, B.M., 2011, "Blow off mechanism of two dimensional bluff body stabilized turbulent premixed flames in a prototypical combustor". Combustion and Flame, Vol. 158, No 7, pp. 1358-1371.

Cheng, R.K., 1984, "Conditional sampling of turbulence intensities and Reynolds stress in premixed turbulent flames". Combustion Science and Technology, Vol. 41, pp.109-142.
Cheng, R.K., Shepherd, I.G. and Gokalp, I., 1989, "A comparison of the velocity and scalar spectra in premixed turbulent flames". Combustion and Flame, Vol.78, No 2, pp. 205-221.

Cheng, R.K. and Shepherd, I.G., 1991, "The influence of burner geometry on premixed turbulent flame propagation". Combustion and Flame, Vol. 85, No 1-2, pp. 7-26.

Dourado, W.M.C., 2003, "Desenvolvimento de um método numérico em malhas não estruturadas híbridas para escoamentos turbulentos em baixo número de Mach: aplicação em chama propagando-se livremente e esteiras inertes e reativas". Tese (Doutorado), Instituto Tecnológico de Aeronáutica, São José dos Campos.

Eriksson, P., 2007, "The Zimont TFC model applied to premixed bluff body stabilized combustion using four different RANS turbulence models". Proceedings of ASME GT2007, ASME Turbo Expo 2007, Vol. 2, pp. 353-361, Montreal.

Frendi, A., Skarath, G. and Tosh, A., 2004, "Prediction of noise radiated by flow over a smooth square cylinder". Proceedings of the 1Oth AIAA/CEAS Aeroacoustics Conference, Manchester, UK.

Fureby, C., Tabor, G., Weller, H.G. and Gosman, A.D., 1997, "A comparative study of subgrid scale models in homogeneous isotropic turbulence". Physics of fluids, Vol. 9, No 5, pp. 1416-1429.

Ge, H.W., Zhu, M., Chen, Y. and Gutheil, E., 2007, "Hybrid unsteady RANS and PDF method for turbulent non-reactive and reactive flows". Flow, Turbulence and Combustion. Vol. 78, No 2, pp. 91-109. 
Ghosal, S., Lund, T., Moin, P. and Akselvoll, K., 1995, "A dynamic localization model for large-eddy simulation of turbulent flows". Journal of Fluid Mechanics, Vol. 286, pp. 229-255.

Jayatilleke, C., 1969, "The influence of prandtl number and surface roughness on the resistance of the laminar sublayer to momentum and heat transfer". Prog. Heat Mass Transfer, Vol. 1, pp. 193-321.

Kerstein, A.R., 1989, "Linear-eddy modeling of turbulent transport II. Application to shear layer mixing", Combustion and Flame, Vol. 75, No 3-4, pp. 397-413.

Kiel, B., Garwick, L.K., Gord, J.G., Miller, J. and Lynch, A., 2007. "A detailed investigation of bluff body stabilized flames". AIAA 2007168, Proceedings of the 45th AIAA Aerospace Sciences Meeting and Exhibit 8 - 11 January 2007, Reno, Nevada.

Lovett, J.A., Brogan, T.P., Philippona, D.S., Keil, B.V. and Thompson, T.V., 2004, "Development Needs for Advanced Afterburner Designs", Proceedings of the 40th AIAA/ASME/SAE/ASEE Joint Propulsion Conference \& Exhibit, Fort Lauderdale, Florida.

Manickam, B., Franke, J., Muppala, S.P. and Dinkelacker, F., 2012 "Large-eddy Simulation of Triangular-stabilized Lean Premixed Turbulent Flames: Quality and Error Assessment". Flow, Turbulence and Combustion. Vol. 88, No 4, pp. 563-596.

Menon, S., McMurtry, P.A. and Kerstein, A.R., 1993, "A linear eddy mixing model for large eddy simulation of turbulent combustion", In: Galperin, B. and Orszag, S. (Eds.), "Large Eddy Simulation of Complex Engineering and Geophysical Flows", Cambridge University Press, Cambridge, pp. 287-314.

Park, N.S. and Ko, S.C., 2011, "Large eddy simulation of turbulent premixed combustion flow around bluff body". Journal of Mechanical Science and Technology, Vol. 25, No 9, pp. 2227-2235.

Peters, N., 2000, "Turbulent Combustion", Cambridge University Press, Cambridge.

Pope, S.B., 2000, "Turbulent Flows”. Cambridge University Press, Cambridge.

Porumbel, I. and Menon S., 2006, "Large Eddy Simulation of Bluff Body Stabilized Premixed Flame", AIAA 2006-152.

Sanquer, S., 1998, "Experimental Study of a Bluff Body Wake, in Presence of Combustion, in Fully Developed Turbulent Channel Flow. Turbulence Scales and Critical Analysis of Transport and Combustion
Models", Ph.D. Thesis, Université de Poitiers, Potiers.

Saghafian, M., Stansby, P.K., Saidi M.S. and Apsley, D.D., 2003, "Simulation of turbulent flows around a circular cylinder using nonlinear eddy viscosity modelling: steady and oscillatory ambient flows". Journal of Fluids and Structures, Vol. 17, No 8, pp. 1213-1236.

Sjunesson, A., Henriksson, R. and Lofstrom, C., 1992, "Cars measurements and visualization of reacting flows in bluff body stabilized flame". AIAA-92-3650.

Sjunnesson, A., Olovsson, S. and Sjoblom, B., 1991, "Volvo Flygmotor internal report", VFA9370-308.

Tabor, G. and Weller, H.G., 2004, "Large eddy simulation of premixed turbulent combustion using Xi flame surface wrinkling model". Flow, Turbulence and Combustion, Vol. 72, pp. 1-28.

Tabor, G.R. and Baba-Ahmadi, M.H., 2010, "Inlet conditions for large eddy simulation: a review". Computers \& Fluids, Vol. 39, No 4, pp. 553-567.

Veynante, D., 2006, "Large eddy simulation of turbulent combustion". Conference on Turbulence and Interaction, Vol. TI 2006, p. 20, Porquerolles, France.

Weller, H.G., Marooney, C.J. and Gosman, A.D., 1990, "A new spectral method for calculation of the time varying area of laminar flame in homogeneous turbulence". Proceedings of the 23rd Symposium (International) on Combustion, The Combustion Institute, Vol. Twenty-third Symposium, pp. 629-636.

Weller, H.G., 1993, "The development of a new flame area combustion model using conditional averaging". Thermo-Fluids Section Report TF 9307, Imperial College of Science, Technology and Medicine, London.

Weller, H.G., Tabor, G., Gosman, A. and Fureby, C., 1998, "Application of a flame-wrinkling les combustion model to a turbulent mixing layer", Symposium (International) on Combustion, The combustion Institute, Vol. 27, No 1, pp. 899-907.

Yoshizawa, A. and Horiuti, K., 1985, "A statistically-derived subgridscale kinetic energy model for the large-eddy simulation of turbulence flows". Journal of Physical Society of Japan, Vol. 54, pp. 2834-2839.

Zimont, V.L. and Lipatnikov, A.N., 1995, "A numerical model of premixed turbulent combustion of gases". Chemical Physics Report, Vol. 14, No 7, pp. 993-1025. 